\title{
Una Introducción a la Derivada desde la Variación y el Cambio: resultados de una investigación con estudiantes de primer año de la universidad
}

\author{
Introduction to Derivative from Variation and Change: research results \\ with first year university students
}

\author{
Silvia Vrancken ${ }^{*}$ \\ Adriana Engler ${ }^{* *}$
}

\begin{abstract}
Resumen
El estudio del cálculo juega un rol importante cuando es necesario cuantificar o medir algún fenómeno. Sin embargo, en el sistema educativo se han priorizado, en general, procesos de construcción formales y aspectos algorítmicos. Buscando dar respuesta a esta problemática se inició una investigación, abordando el estudio de la derivada. El trabajo se enmarcó en la línea del Pensamiento y Lenguaje Variacional, que estudia la articulación entre la investigación y las prácticas sociales que dan vida a la matemática de la variación y el cambio en los sistemas didácticos. En el contexto de una ingeniería didáctica, se diseñó una secuencia que se llevó al aula con alumnos de Matemática II de la carrera Ingeniería Agronómica. Las actividades permitieron analizar diversos escenarios de variación (qué magnitudes cambian, cómo y cuánto cambian), caracterizar variaciones entre las magnitudes, a través del cálculo de razones de cambio, y explorar cómo la pendiente de una curva se relaciona con la razón de cambio. Su presentación, desde diferentes representaciones, favorece el desarrollo de uno de los procesos cognitivos implicados en el pensamiento matemático, el de visualización. En este artículo se describen brevemente los aspectos teóricos y metodológicos que fundamentan el diseño de la secuencia y se presentan algunos resultados obtenidos de su implementación a partir de la observación de las actividades desarrolladas por los alumnos. La propuesta logró motivar a los alumnos y movilizar sus concepciones. La discusión de los resultados obtenidos favorece la optimización de la secuencia.
\end{abstract}

Palabras-Claves: Pensamiento Variacional. Derivada. Enseñanza. Aprendizaje.

\begin{abstract}
The study of calculus plays an important role when it is necessary to quantify or measure any phenomenon. However, in general, in the educational system, formal construction processes and algorithmic aspects have been prioritized. Seeking to answer this problem, an investigation addressing the study of the derivative was started. The work was framed from the perspective of Variational Thought and Language, which studies the relationship between research and social practices that rules the mathematics of change and variation in educational systems. In a didactic engineering context, a sequence was designed and presented in a Mathematics II class attended by Agricultural Engineering students. The activities made it possible to analyse different scenarios of variation (which magnitudes change, how and how much they change), characterize variations between magnitudes,
\end{abstract}

\footnotetext{
Profesora en Matemática y Magíster en Didácticas Específicas por la Universidad Nacional del Litoral. Docente de la Facultad de Ciencias Agrarias de la Universidad Nacional del Litoral, Esperanza, Santa Fe, Argentina. Dirección Postal: Kreder 2805. (3080), Esperanza, Santa Fe, Argentina. E-mail: svrancke@fca.unl.edu.ar

Magíster en Educación Psico Informática. Estudiante del Doctorado en Matemática Educativa, por el Instituto Politécnico Nacional, México. Docente de la Facultad de Ciencias Agrarias de la Universidad Nacional del Litoral, Esperanza, Santa Fe, Argentina. Dirección Postal: Kreder 2805. (3080) Esperanza, Santa Fe, Argentina. E-mail: aengler@fca.unl.edu.ar
} 
through the calculation of rates of change, and explore how the slope of a curve is related to the rate of change. Its presentation from different representations contributes to the development of one of the cognitive processes involved in mathematical thinking: visualization. In this article, the theoretical and methodological aspects that support the design of the sequence are briefly described, and some results of its implementation from the observation of the activities developed by the students are presented. The proposal managed to motivate students. The discussion of the results will contribute to the optimization of the sequence.

Key words: Variational Thinking. Derivative. Teaching. Learning.

\section{Introducción}

La matemática juega un rol importante cuando es necesario cuantificar o medir cualquier fenómeno y las variaciones que se producen. El cálculo tiene reconocida su importancia porque permite encontrar las leyes que describen esos cambios, medirlos y predecirlos. En particular, la derivada permite cuantificar, describir y pronosticar la rapidez de la variación en fenómenos de la naturaleza o de la práctica.

Sin embargo, en el sistema educativo se han priorizado, en general, procesos de construcción y validación formales así como los aspectos algorítmicos. Artigue (1995) manifiesta que la enseñanza universitaria del cálculo, aunque tenga otras ambiciones, tiende a centrarse en una práctica algorítmica, o en la aplicación de métodos tradicionales de demostración matemática.

De esta manera, si bien se logra que los estudiantes deriven, integren o calculen límites elementales, no son capaces de asignar un sentido más amplio a las nociones involucradas. $\mathrm{Al}$ respecto, Dolores (2007b, p. I) expresa que los estudiantes “[...] difícilmente logran reconocer las ideas asociadas al concepto de derivada en la resolución de problemas elementales sobre variación y cambio a pesar de que en los problemas de este tipo se encuentra la esencia de este concepto".

Esto parece ocasionar consecuencias negativas, más aún cuando los que aprenden son estudiantes que, para el ejercicio de su profesión, necesitan conocimientos y habilidades que les permitan resolver problemas reales. Es el caso de los alumnos de carreras de ingeniería y, en particular, de ingeniería agronómica, contexto en el que se desarrolló este trabajo.

Buscando dar respuesta a esta problemática se planteó una investigación que abordó el estudio de la derivada. La pregunta inicial fue qué y cómo enseñar de manera de lograr que el alumno pueda comprender y dar sentido a los conceptos y procedimientos, de manera de contribuir al desarrollo de su pensamiento y a la construcción de conocimiento matemático. 


\section{Marco de referencia}

La base teórica en la que se fundamentó nuestro trabajo es la del Pensamiento y Lenguaje Variacional. Como parte del pensamiento matemático avanzado, el pensamiento variacional comprende las relaciones entre la matemática de la variación y el cambio y los procesos de pensamiento. Tiene en cuenta, además, la necesidad de analizar la relación de los saberes con prácticas socialmente compartidas y con sentidos y significados extra matemáticos.

El pensamiento y lenguaje variacional estudia los fenómenos de enseñanza, aprendizaje y comunicación de saberes matemáticos propios de la variación y el cambio en el sistema educativo y en el medio social que le da cabida. Hace énfasis en el estudio de los diferentes procesos cognitivos y culturales con que las personas asignan y comparten sentidos y significados utilizando diferentes estructuras y lenguajes variacionales (CANTORAL et al., 2003, p. 185).

Su objeto principal de estudio son los fenómenos de cambio y su entendimiento. En este contexto, un concepto primordial es el cambio, modelado matemáticamente mediante la diferencia. Las diferencias dan cuenta de cuánto cambia la variable en un proceso de variación. En este sentido, son el elemento central de todo el cálculo, por eso a esta parte de la matemática se la conoce como matemática de la variación y el cambio.

La derivada refleja, con gran precisión, una de las propiedades esenciales de los fenómenos de la realidad: la rapidez de la variación. Está relacionada con tres nociones fundamentales: el cambio, la razón promedio de cambio y la razón instantánea de cambio.

La incorporación de elementos variacionales y el otorgamiento de significado a los distintos elementos relacionados a la variación en estudio favorecerán la construcción de la derivada. En un sentido más amplio, influirán positivamente en el desarrollo del pensamiento variacional de los alumnos, y, también, de su lenguaje variacional, en tanto sean capaces de comunicar sus ideas (TESTA, 2004; DOLORES, 2007b; BUENDÍA; ORDÓÑEZ, 2009).

Otro aspecto que resulta esencial en el desarrollo del pensamiento y en la producción de conocimiento matemático es el empleo de nociones asociadas a los registros numérico, gráfico, algebraico y verbal. Duval $(1998,2008)$ señala que la comprensión integral de un objeto está basada en la coordinación de al menos dos sistemas de representación pertenecientes a registros diferentes. En general, las tareas de conversión entre diferentes sistemas de representación son minimizadas y eso produce limitaciones en la comprensión y en el desarrollo de uno de los estilos de pensamiento, el visual.

Diversas investigaciones en Educación Matemática proporcionan ejemplos sobre problemas de aprendizaje y el papel de la visualización en la comprensión del cálculo 
(CANTORAL et al., 2003). Si bien se reconoce su importancia a fin de favorecer la comprensión matemática, existen numerosas investigaciones que muestran la gran resistencia de los alumnos a visualizar. Muchas de las dificultades del cálculo se superarían si se enseñara a los estudiantes a interiorizar las connotaciones visuales de los distintos conceptos.

En el estudio de las circunstancias que permiten construir conocimiento no se pueden dejar de lado aspectos sociales y culturales. Es en el seno de la organización social donde se reconstruyen significados de la matemática como recurso para aceptar determinado conocimiento matemático. El conjunto de actividades que realiza el alumno en una situación concreta permite otorgar significado a los conceptos y construir conocimiento matemático. Cantoral y Montiel (2001, p. v) expresan:

Se ha puesto en evidencia que los alumnos construyen conocimiento con cierta
independencia del discurso matemático de la enseñanza. Con frecuencia, construyen
explicaciones inadecuadas e inclusive erróneas desde un punto de vista matemático,
a la vez que descubren profundas relaciones entre piezas del saber matemático, sin
que eso haya sido parte explícita de su enseñanza.
Consideramos que estos conocimientos son el fruto de la interacción con su entorno:
con sus compañeros, con sus historias de vida o con su ambiente académico y
cultural, entre otros.

Esto hace que la actividad matemática no pueda ser abordada de una manera sencilla. Su enseñanza debe contribuir a que el estudiante desarrolle sus potencialidades y logre la formación de un pensamiento productivo, creador y científico. Es importante crear en el estudiante la necesidad de aprender y generar un ambiente donde se posibilite y se motive la exploración del significado personal de los conceptos.

En este contexto se planteó, como objetivo de investigación, indagar las nociones, relacionadas a la derivada, que construyen los estudiantes cuando interactúan con actividades articuladas en torno a la idea de variación y cambio, que favorecen el tratamiento y la articulación de diferentes sistemas de representación.

Dada la complejidad de los elementos implicados en los procesos de enseñanza y aprendizaje, resultó necesario un marco de indagación que intente comprender los procesos en profundidad. Se recurrió a la metodología de la ingeniería didáctica, lo que llevó a la elaboración de una secuencia didáctica. En su diseño y puesta en práctica se tuvieron en cuenta las fases correspondientes a la metodología utilizada: análisis preliminares, concepción de la ingeniería, experimentación y análisis a posteriori. 


\section{El diseño de la secuencia}

Los estudios preliminares permitieron conocer el origen y evolución histórica del saber en juego, llevaron a revisar el estado actual de la enseñanza del tema y al mismo tiempo analizar las dificultades y obstáculos que su aprendizaje genera.

Desde sus orígenes el cálculo se caracterizó por tener un componente fundamentalmente visual e intuitivo, que se mantuvo durante los siglos siguientes, en interacción constante con problemas geométricos y físicos. El análisis de la variación en los fenómenos dinámicos es lo que condujo al estudio de la derivada y al desarrollo del cálculo.

El análisis de libros de texto, de uso común en nuestro entorno, y de los diseños curriculares para la escuela secundaria, mostró que la enseñanza de las funciones y del cálculo diferencial no se corresponde generalmente con sus orígenes, privilegiando en cambio aspectos lógicos formales. Se dedica mucho tiempo a la enseñanza de algoritmos, dejando de lado la formación de ideas variacionales. Si bien la introducción de ideas fundamentales del cálculo está planteada desde la escuela secundaria, esto no se alcanza en la práctica, por lo menos en la mayoría de las instituciones de nuestro entorno, por lo que los alumnos tienen su primer contacto con la matemática del cambio en la universidad.

La aplicación a nuestros alumnos de un cuestionario, previo al inicio del desarrollo de las nociones de cálculo, reveló las deficiencias que presentan con respecto a nociones variacionales básicas, necesarias para la comprensión y utilización de conceptos fundamentales como el de derivada. Los problemas detectados para el tratamiento y conversión entre registros, especialmente para interpretar información presentada gráficamente, dificultan la posibilidad de que los alumnos visualicen los conceptos o determinados aspectos de los mismos.

Los estudios realizados llevaron a plantear la necesidad de un primer acercamiento visual e intuitivo a los conceptos del cálculo diferencial partiendo del estudio de la variación, atendiendo a tres de sus aspectos básicos: el cambio, la razón media de cambio y la razón instantánea de cambio. En estas nociones se encuentra el origen de la derivada. La idea fue, siguiendo a Dolores (2007a, p. 198):

[...] ubicar como eje rector de todo el curso de Cálculo Diferencial al estudio de la variación, de modo que la derivada no sea un concepto matemático abstracto sino un concepto desarrollado para cuantificar, describir y pronosticar la rapidez de la variación en fenómenos de la naturaleza o de la práctica.

En el trabajo interesó entender y explicar la forma en que la representación de la variación interviene en la construcción del concepto de derivada. Testa (2004) expresa que 
para dar significado variacional a la derivada, es necesario significar previamente cada elemento que varía. Si existe la derivada de una función $\mathrm{f}$ en un real $a$, será $\mathrm{f}^{\prime}(\mathrm{a})=\lim _{\mathrm{x} \rightarrow \mathrm{a}} \frac{\mathrm{f}(\mathrm{x})-\mathrm{f}(\mathrm{a})}{\mathrm{x}-\mathrm{a}}$. Este límite brinda información de cómo y cuánto varía la función $f$ en un entorno del real $a$. Pero, ¿a qué tipo de variación de la función $f$ se refiere? A una variación relacionada con la diferencia $f(x)-f(a)$, pero, ¿es posible significar esta variación si previamente no se da significado a $f(x)$ y $f(a)$ ?

Estos elementos son los que caracterizan básicamente al pensamiento variacional y que en la secuencia se pretendió analizar.

Para el diseño de las actividades se tuvieron en cuenta las ideas desarrolladas por Wenzelburger (1993), Dolores (1999, 2007b) y Azcárate et al. (1996), quienes manifiestan la necesidad de partir de las concepciones previas de los alumnos acerca de la velocidad, utilizar las gráficas de las funciones para visualizar ideas, en especial la de razón de cambio media como pendiente de una recta.

Se desarrollaron actividades que permiten analizar distintos escenarios de variación en el contexto de la física (qué magnitudes cambian, cuánto cambian, cómo cambian), que llevan a la necesidad de caracterizar variaciones entre magnitudes, a través del cálculo de razones de cambio y que posibilitan, a su vez, la exploración de la manera en que estas razones de cambio están relacionadas con la medida de una pendiente. Se alternan los registros verbal, gráfico, numérico y analítico, requiriendo el tratamiento y conversión entre los mismos.

En la primera parte de la secuencia, de orientación hacia la construcción de la derivada, se plantearon situaciones que llevaron a trabajar nociones relacionadas con la razón de cambio media. En primer lugar, en el contexto del movimiento rectilíneo uniforme, buscando relacionar la razón de cambio media con la pendiente de la recta. Se trabajaron luego situaciones donde la velocidad no es constante. Esto dio lugar a pensar sobre la necesidad de determinar la velocidad en cada instante. Se planteó la imposibilidad de resolver el problema mediante la razón de cambio media.

Esto sirvió de motivación para introducir la segunda parte, de construcción de la derivada, intentando encontrar solución al problema. Se plantearon, en primer lugar, las aproximaciones numéricas a la velocidad en un instante, proponiendo la reducción de los intervalos. Desde el punto de vista geométrico, se exploró la relación de la velocidad media con la pendiente de la recta secante y de la aproximación de la velocidad instantánea con la pendiente de la recta tangente. Se trabajaron las aproximaciones, buscando que descubran la necesidad de realizar el paso al límite para calcular la velocidad instantánea. 


\section{Implementación de la secuencia}

La secuencia se llevó al aula con los alumnos cursantes de Matemática II de la carrera Ingeniería Agronómica de la Facultad de Ciencias Agrarias de la Universidad Nacional del Litoral. Los mismos habían aprobado o regularizado Matemática I, asignatura en la cual se desarrollan los contenidos referentes a funciones.

Siguiendo el programa de la asignatura los alumnos, ya habían repasado los contenidos referidos a números y la recta real, y estudiado los temas límite y continuidad.

En la clase previa a la implementación de la secuencia, resolvieron actividades relacionadas al comportamiento variacional de las funciones. Se repasaron distintas maneras de expresar los cambios, cualitativamente, describiéndolos, cuantitativamente, a través de tablas, simbólicamente, utilizando las notaciones correspondientes.

Para el desarrollo específico se dedicaron cinco horas reloj distribuidas en tres clases. Los primeros minutos de cada clase se dedicaron a repasar lo desarrollado en la anterior.

Teniendo en cuenta que la construcción del conocimiento se realiza dentro de las prácticas sociales, se planificó una modificación importante del ambiente de clase, introduciendo una dinámica de interacción social. Se decidió privilegiar las prácticas compartidas, de manera de proporcionar el ámbito para contrastar significados, ya sea en grupos pequeños como en la discusión de la clase completa.

En primera instancia, los alumnos resolvieron las actividades en grupos de a dos. El profesor apoyó a los equipos, atendiendo las preguntas pero intentando que ellos mismos encontraran las respuestas. En la última parte de cada clase se hizo una discusión grupal, revisando las distintas actividades. En esta instancia el profesor intervino, pidiendo justificaciones, introduciendo contradicciones, planteando sus argumentos sin imponerlos. Todo esto fue llevando a la construcción de conocimiento, que fue aprovechado para la formalización del trabajo realizado, teniendo presente en todo momento los aspectos variacionales que son la base de las distintas actividades y relacionando las representaciones numéricas, gráficas y analíticas de todos los conceptos involucrados.

\section{Análisis de la secuencia y valoración de resultados}

En este apartado se analizan algunos aspectos que surgieron de la implementación de la propuesta, resaltando aquellos que implican el desarrollo del pensamiento variacional, 
específicamente los aspectos variacionales de las distintas actividades y el tratamiento y conversión entre distintas representaciones.

Distintos elementos dan cuenta del desarrollo del pensamiento variacional. Estudiar la variación de un sistema o cuerpo significa ejercer nuestro entendimiento para conocer cómo y cuánto cambia el sistema o cuerpo dado. Es en este sentido que nos referimos a los argumentos de tipo variacional. Decimos que una persona utiliza o comunica argumentos y estrategias variacionales cuando hace uso de maniobras, ideas, técnicas, o explicaciones que de alguna manera reflejan y expresan el reconocimiento cuantitativo y cualitativo del cambio en el sistema u objeto que se está estudiando (CANTORAL; MOLINA; SÁNCHEZ, 2005).

Para el análisis se tuvieron en cuenta las producciones escritas de 23 equipos de trabajo. La selección de estos equipos se hizo teniendo en cuenta que sus integrantes cursaran por primera vez Matemática II, hayan asistido a todas las clases y hayan mantenido la conformación del grupo de trabajo durante todas las sesiones. Se consideraron, también, los registros de las observaciones del docente y de un colaborador presente en el aula durante el desarrollo de la secuencia. Esto fue un aporte valioso para describir los distintos momentos de interacción, qué dudas surgieron a lo largo de la resolución de las actividades, cómo respondían los alumnos a las preguntas del docente.

Se presentan los trabajos de algunos equipos y, a partir de los mismos, se comentan los principales logros y dificultades. Se transcriben, también, tramos de entrevistas realizadas la semana posterior al desarrollo de la secuencia, con la intención de obtener más información sobre los procesos y razonamientos que habían seguido en sus respuestas, profundizando de esta manera en sus concepciones.

En la primera actividad se presentó una gráfica espacio-tiempo que describe el movimiento de un auto. La misma se tomó de un problema propuesto en Azcárate et al. (1996), adaptando la gráfica y las preguntas a nuestro contexto. Coincidiendo con lo que proponen los autores, se parte de una situación que los alumnos pueden imaginar. La lectura de la gráfica permite obtener datos sobre el movimiento a partir de los cuales es posible calcular las velocidades medias para distintos intervalos de tiempo. Se plantea, también, el cálculo de la velocidad en un instante. Se esperaba que los alumnos puedan descubrir, ya que se trata de un movimiento con tramos de velocidad constante, que coincide con la velocidad promedio en el intervalo que lo contiene. En la Figura 1 se presenta la resolución de un equipo. 


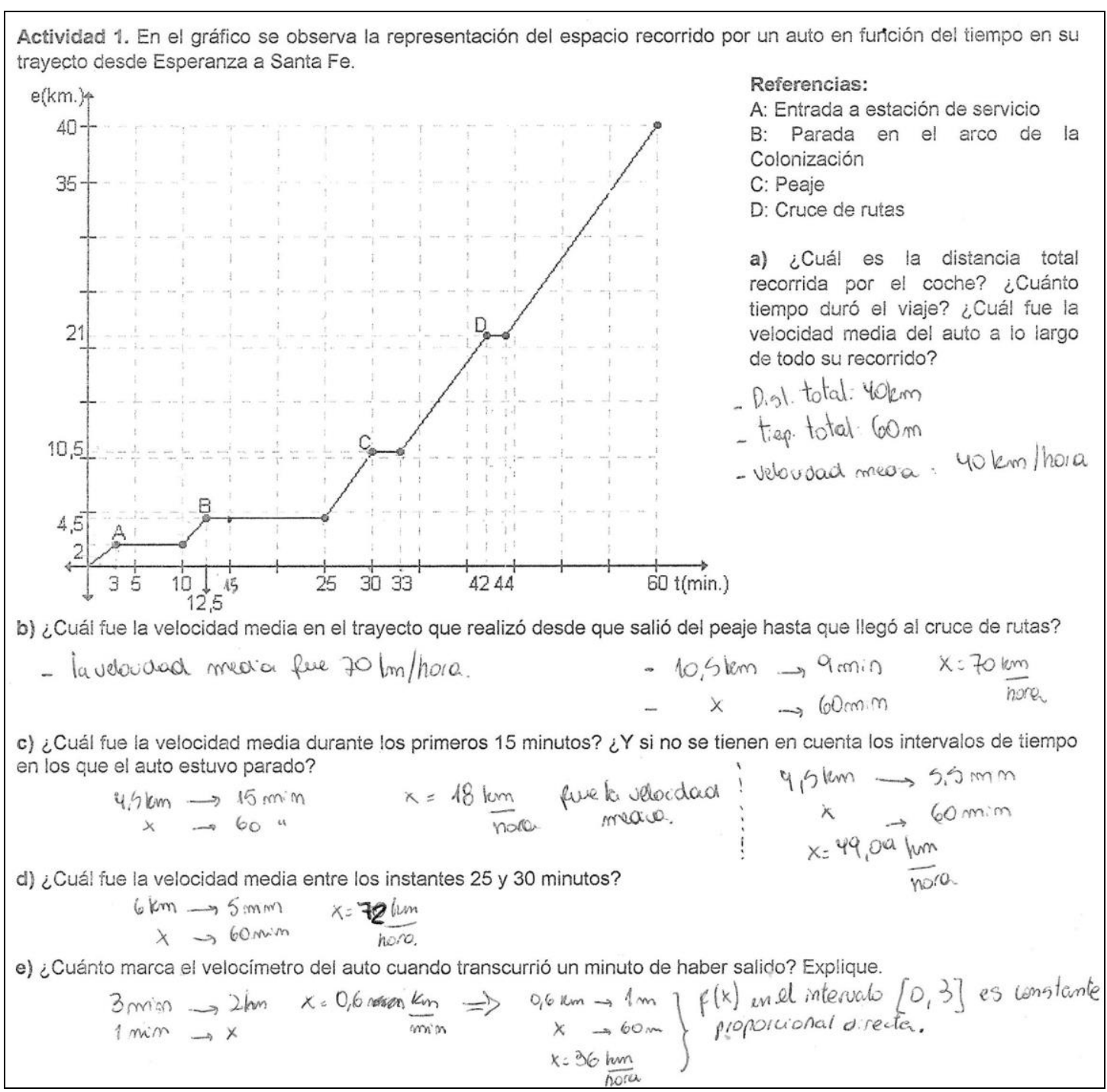

Figura 1 - Resolución de una situación de movimiento con tramos de velocidad constante Fuente: datos de la investigación

Para la resolución de esta actividad, los alumnos recurrieron, en general, a sus conocimientos previos, trabajando la idea de velocidad como espacio sobre tiempo. Se observó que identificaron la representación gráfica con una situación de movimiento con tramos de velocidad constante. La mayor dificultad fue la interpretación y diferenciación de las nociones de velocidad media y velocidad instantánea. En este sentido, sus concepciones sobre aspectos de cinemática se revelaron bastante pobres. Al ser orientados acerca del significado de ambas nociones, apelaron a procedimientos relacionados con la proporcionalidad directa, determinando el espacio recorrido en la unidad de tiempo.

Con respecto a los registros, esta actividad exige obtener los datos desde el registro gráfico y trabajarlos numéricamente. Los alumnos no presentaron dificultades, excepto en el inciso b. En este apartado, es el único en el que no se dan explícitamente los valores de la 
variable independiente, sino que los alumnos debían obtenerlos a partir de la interpretación de los puntos correspondientes a las distintas referencias.

A pesar de los inconvenientes, la actividad cumplió con nuestro objetivo de, a partir de nociones que ellos manejan, o tareas que pueden realizar, comenzar a construir otras nuevas.

En las dos actividades siguientes se presentó una función definida algebraicamente, cuyo objetivo principal fue que los alumnos trabajen con la velocidad media, a partir de la obtención de las diferencias (incrementos) y de los cocientes entre estas diferencias. Para su diseño se consideraron las ideas propuestas por Dolores (1999) y Wenzelburger (1993). Se esperaba que su resolución condujera al reconocimiento del tipo de funciones, o representaciones gráficas, que dan lugar a razones de cambio constantes y aquellas que llevan a razones de cambio variables. Requiere la traducción del registro analítico al numérico y gráfico además de interpretar lo realizado en el registro verbal.

Se presenta la resolución de un equipo de la actividad 2 (Figura 2).

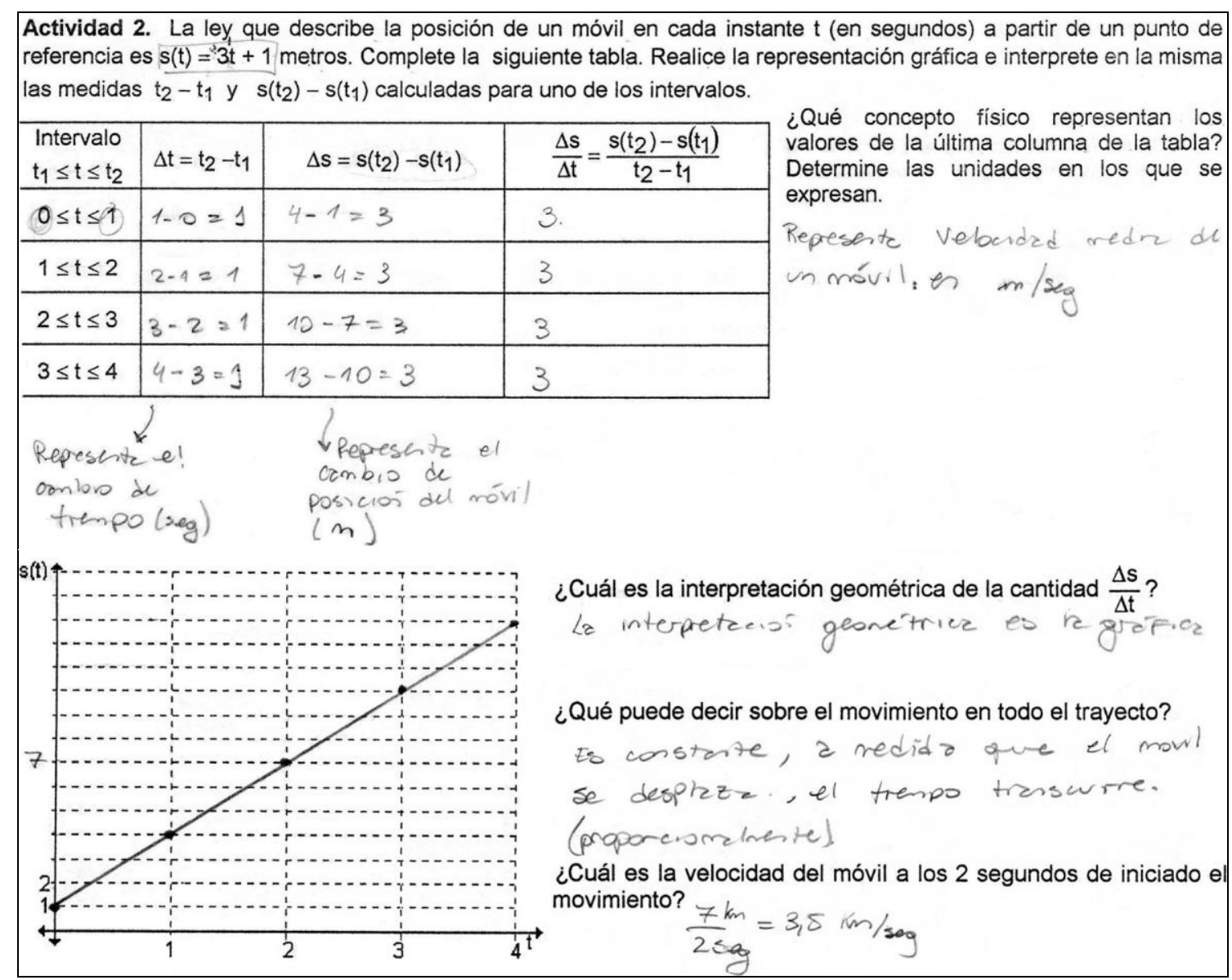

Figura 2 - Resolución de una situación de movimiento con velocidad constante Fuente: datos de la investigación

Más de la mitad de los alumnos respondió de manera similar al trabajo presentado. Los alumnos tuvieron oportunidad de identificar la diferencia como la operación que permite medir los cambios. Además, asociar el cociente de la última columna, que corresponde a la 
razón de cambio media, con la velocidad media. Esta reflexión la podemos inducir a partir de las respuestas a preguntas como: ¿Qué concepto físico representan los valores de la última columna de la tabla?, y también: ¿Qué puede decir sobre el movimiento en todo el trayecto?

Relacionaron el tipo de movimiento de acuerdo a la velocidad, aunque no lo lograron expresar correctamente. El hecho de que observaran que los cambios son constantes es muy importante desde nuestro enfoque variacional. Los movimientos están determinados por los cambios y el comportamiento de los cambios es el aspecto esencial de la variación.

Otra parte importante manifestó que el movimiento es rectilíneo uniforme. En este caso, los alumnos recurrieron a sus conocimientos previos para responder.

Se observó que, a partir de la actividad, los estudiantes dieron un significado a la razón de cambio media y caracterizaron una situación de razón de cambio constante, trabajando en distintos registros. La identificaron verbalmente con un movimiento de velocidad constante, con los cálculos realizados en el registro tabular y gráficamente con la gráfica de la recta que corresponde al movimiento.

Otro de los aspectos importantes de esta actividad fue la interpretación geométrica del cociente. A pesar de que trajo bastantes dificultades, como se observa en el primer trabajo (Figura 2), varios equipos pudieron relacionarlo con la pendiente de la recta que representa el movimiento (Figura 3).

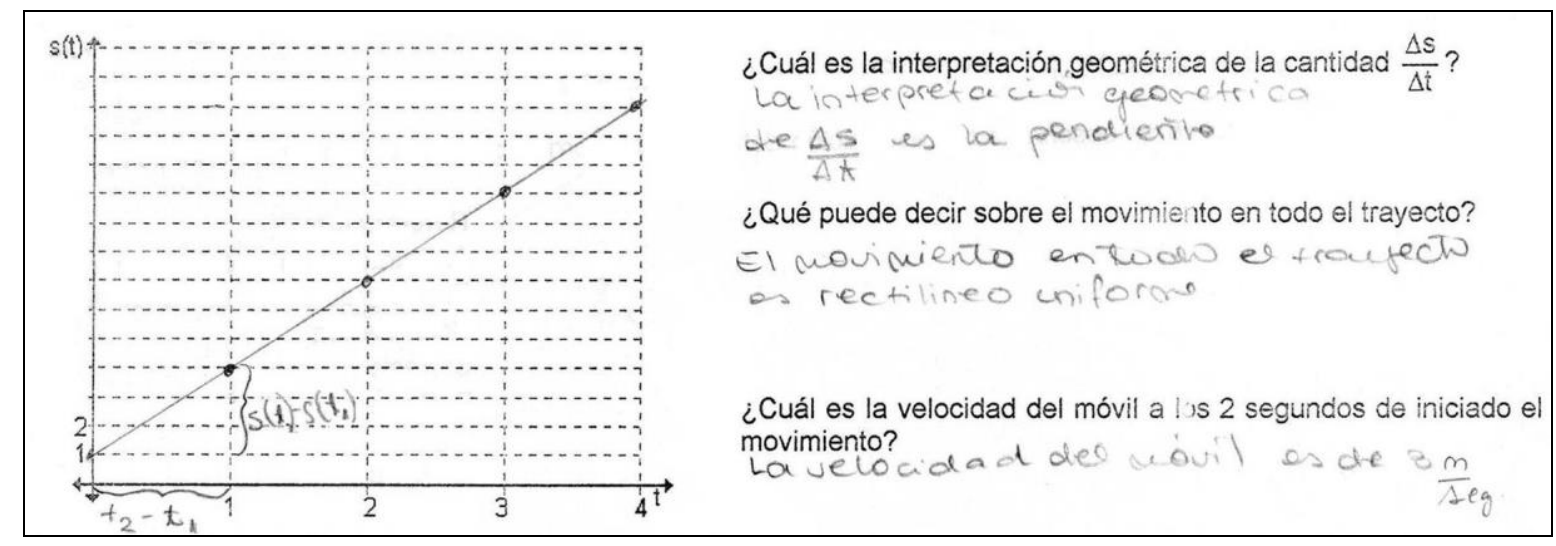

Figura 3 - Interpretación gráfica en una situación de velocidad constante Fuente: datos de la investigación

Algunos grupos realizaron la interpretación de las medidas $t_{2} \quad t_{1} \quad \mathrm{y} \quad \boldsymbol{s}\left(t_{2}\right) \quad \boldsymbol{s}\left(t_{1}\right)$ en la gráfica, asociando las magnitudes involucradas en la situación de cambio con longitudes de segmentos. Esto es muy importante, teniendo en cuenta la necesidad de la conversión entre registros para una verdadera comprensión.

Con respecto a la última pregunta ¿cuál es la velocidad del móvil a los 2 segundos de iniciado el movimiento?, su respuesta trajo nuevamente bastantes dificultades, recurriendo, en 
general, al cálculo de la velocidad media durante los dos primeros segundos. Sin embargo, obligó a los alumnos a comenzar a cuestionarse sobre distintos tipos de velocidad.

Al ser consultados en la entrevista, varios alumnos manifestaron comprensión de la situación, interpretando correctamente lo trabajado en clase y lo desarrollado durante la instancia de institucionalización. Se transcribe un tramo de la entrevista realizada a Vanesa.

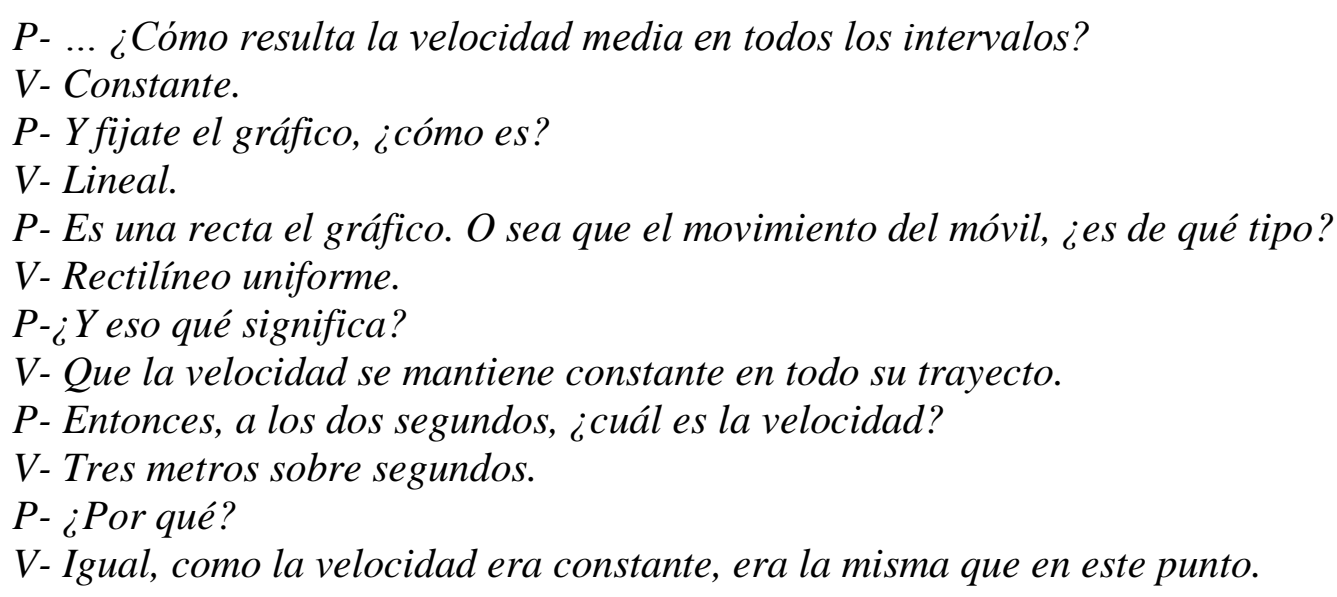

Se nota claridad en sus observaciones, integrando el trabajo realizado en los distintos registros. Resalta el hecho de poder asociar el tipo de movimiento con la representación gráfica de la trayectoria y poder inferir que, como la velocidad es constante, la velocidad en cualquier instante coincide con la velocidad media en cualquier intervalo.

En la siguiente actividad se presentó una función posición-tiempo que corresponde a un movimiento variado y que agrega la dificultad de cambios de posición negativos. Se presenta la resolución de un equipo (Figura 4).

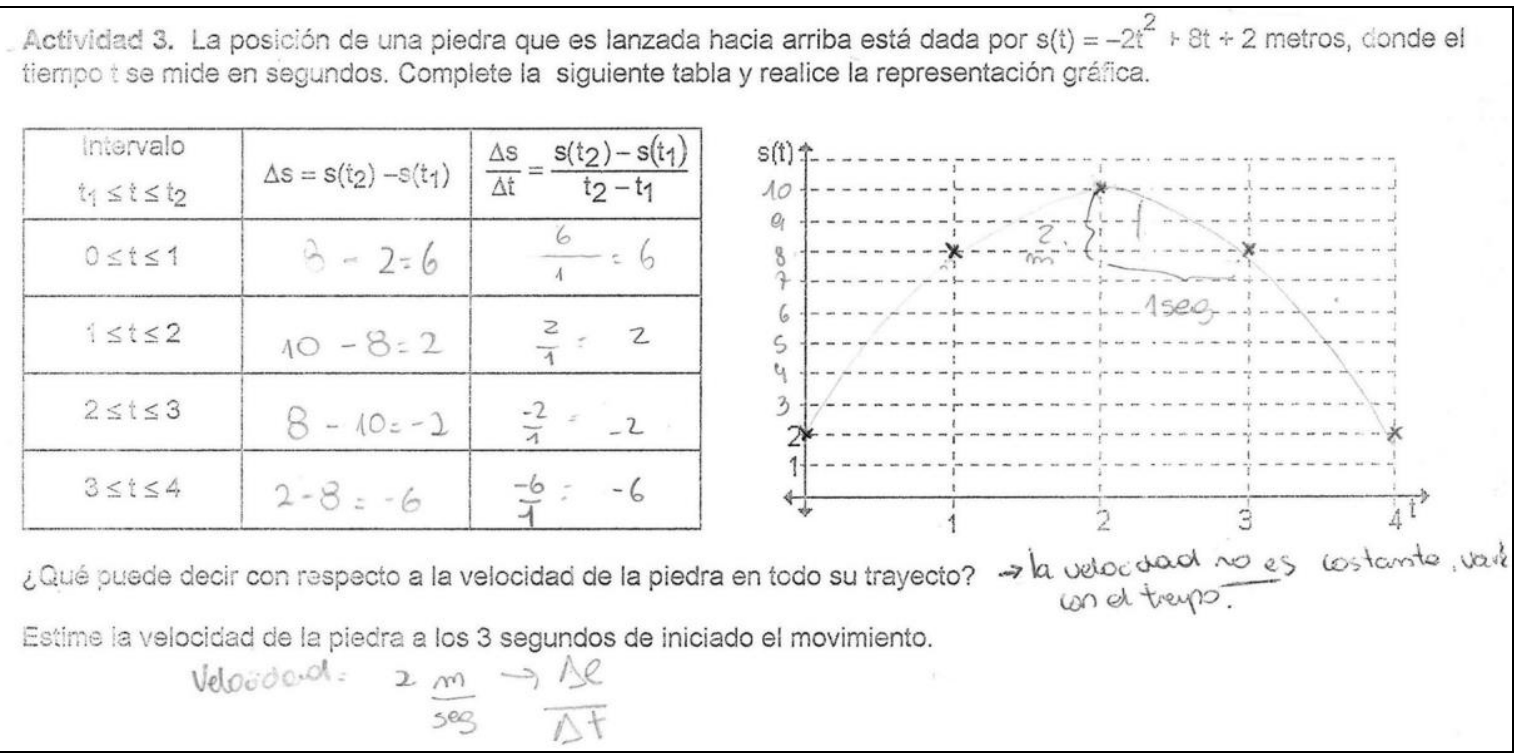

Figura 4 - Resolución de una situación de movimiento con velocidad variable Fuente: datos de la investigación 
La determinación y el análisis de los cambios cuantitativos son fundamentalmente importantes para la conceptualización de la razón de cambio. Con la ayuda del registro tabular los estudiantes determinaron que la variación del tiempo es constante, debido a que la medición siempre se hace cada un segundo, mientras que la variación de posición no es constante, por lo que los cocientes tampoco lo son. Este tratamiento, sumado al análisis de la gráfica, les permitió reconocer el tipo de funciones que dan lugar a razones de cambio variables distinguiéndolas de aquellas que llevan a razones de cambio constantes.

Para estimar la velocidad de la piedra a los 3 segundos, se observó cómo algunos grupos aproximaron la velocidad en el instante, considerando la velocidad media en un intervalo. Algunos lo hicieron numéricamente, planteando el cociente. En la resolución que se presenta en la Figura 4, los alumnos señalaron en el gráfico las variaciones correspondientes, mostrando que consideraron el intervalo [2,3] para aproximar la velocidad.

Algunos de estos aspectos se presentan en la entrevista realizada a Luis.

$P$ - Observando la gráfica, ¿me podés describir cómo es la trayectoria que realizó la piedra?

L- La posición de la piedra va cambiando sería.

$P$ - Sí. ¿Cómo va cambiando?

L- No de manera uniforme sería. Va cambiando en un movimiento que no se puede describir con una recta.

P- Explicame cómo va variando la posición de la piedra.

L- Va a subir hasta cierto punto que va a bajar.

$P-¿$ Hasta qué instante sube la piedra?

L- Hasta el instante dos.

P-Entonces, como podés, relacionando esos conceptos, decir que la velocidad ahí es cero, cómo explicás.

L-Porque la pendiente de la recta tangente ahí es cero.

$P$ - Sí.

L- Nosotros habíamos trazado la recta tangente así, así, así (muestra en distintos puntos) hasta que en un punto la pendiente va a valer cero.

La lectura del tramo de entrevista permite observar varias cosas importantes:

- $\quad$ Describe de una manera que evidencia influencia del lenguaje utilizado por los docentes en las clases, refiriéndose a los cambios.

- Explica adecuadamente el comportamiento de la posición de la piedra. Relaciona el tipo de movimiento con los cambios que no son constantes y con la gráfica que no es una recta.

- Relaciona la velocidad con la pendiente de la recta tangente. Aparece la idea de pendiente variable, importante para una concepción dinámica de la tangente y la formación de 
la noción de dirección de una curva. Las inclinaciones de la recta tangente en los diferentes puntos de una curva dan idea de cuán rápido cambia eso que cambia.

Conclusiones de este tipo son indicadores del desarrollo de estrategias del pensamiento variacional. Son importantes, también, para comprender otros aspectos del comportamiento variacional de una función, como cuándo una función es creciente o decreciente o por qué en determinados puntos alcanza un valor máximo o mínimo.

En la actividad siguiente se dio una función espacio-tiempo definida numéricamente. Las nociones involucradas son, nuevamente, la de velocidad media y velocidad instantánea. Teniendo en cuenta la imposibilidad de conocer la velocidad en un instante a partir de la media ya que el movimiento no es uniforme, se plantea su cálculo utilizando la fórmula de velocidad media. Dado que se llega a una indeterminación, se muestra que no es posible seguir este camino. Sin embargo, se pueden calcular las razones de cambio medias en intervalos cada vez más pequeños de manera de caracterizar más exactamente la razón de cambio en este tipo de fenómenos. Se muestra la resolución de un equipo (Figura 5).

a) $E l$ investigador intentó determinar la velocidad exacta de la partícula en $t=2$ segundos mediante la fórmula
$\frac{\Delta e}{\Delta t}=\frac{e\left(t_{2}\right)-e\left(t_{1}\right)}{t_{2}-t_{1}}$ haciendo coincidir el instante inicial y el final del intervalo, es decir considerando $t_{1}=2$ y $t_{2}=2$. ¿Qué
puede observar sirrealiza este procedimiento?
$\frac{\Delta e}{\Delta t}=\frac{\theta}{\theta} \Rightarrow$ Noxiste.

b) Complete la tabla con los espacios recorridos y las velocidades medias de la partícula en el intervalo [2, t] teniendo en cuenta que los valores de $t$ son los que aparecen en la primera fila de la tabla anterior.

\begin{tabular}{|l|c|c|c|c|c|c|c|}
\hline Intervalo $[2, \mathrm{t}]$ & {$[2 ; 2,1]$} & {$[2 ; 2,2]$} & {$[2,2,3]$} & {$[2,2,4]$} & {$[2 ; 2,5]$} & {$[2 ; 3]$} & {$[2 ; 4]$} \\
\hline Espacio recorrido en el intervalo $[2, t]$ & 0,37 & 0,68 & 0,93 & 1,13 & 1,28 & 1,5 & 4 \\
\hline Velocidad media en el intervalo $[2, t]$ & 3,7 & 3,4 & 3,1 & 2,825 & 2,56 & 1,5 & 2 \\
\hline
\end{tabular}

Según los cálculos realizados, ¿cuál de los valores para la velocidad media es una mejor aproximación de la velocidad

El valor de velocidad mediz que es und mejor Aproximacion a la velocidad en el instante $t=2$ es $3,7([2 ; 2,1])$ YA QUE ES MENOS VARIAGí́N DE TIEMPO TIENE

Figura 5 - Aproximación de la velocidad instantánea en una situación con velocidad variable Fuente: datos de la investigación

Se presentaron muchas dificultades para la resolución de esta actividad, pero las ideas que se buscaban generar surgieron en varios equipos: en los fenómenos que cambian a cada instante, dada la ley del movimiento, es posible calcular la velocidad en determinado intervalo, pero ese resultado no alcanza para determinar el comportamiento preciso de los cambios. Los alumnos reflexionaron sobre el hecho de que la velocidad instantánea no es la misma que la media y por lo tanto no se puede calcular de la misma manera. El planteo en el registro numérico facilitó el cálculo de los espacios recorridos y las velocidades medias. Se sorprendieron ante la forma de cálculo planteada en el primer inciso, haciendo coincidir 
instante inicial con final. Con respecto a la interpretación de la tabla, dada la imposibilidad de usar la estrategia anterior, les pareció natural asignar variaciones de tiempo de manera de acercarse a $\mathrm{t}=2$.

Con esta actividad quedó planteada la imposibilidad de resolver el problema mediante la razón de cambio media. Esto sirvió de motivación para introducir la segunda fase de la secuencia, la de construcción de la derivada, intentando encontrar solución al problema. Para esto, desde el punto de vista geométrico, se exploró la relación de la velocidad media con la pendiente de la recta secante a la gráfica de la función y de la velocidad instantánea con la pendiente de la recta tangente. Se siguieron trabajando las aproximaciones, buscando que descubran la necesidad de realizar el paso al límite para calcular la velocidad instantánea y se trabajaron algebraicamente ambas nociones.

A continuación (Figura 6) se presenta la resolución de parte de la actividad siguiente, en la que se propuso un acercamiento gráfico con una función que describe la posición de una pequeña bola en caída libre. En los primeros incisos los alumnos debieron retomar lo trabajado, determinando las velocidades medias en distintos intervalos y aproximando la velocidad de la bola en un instante.

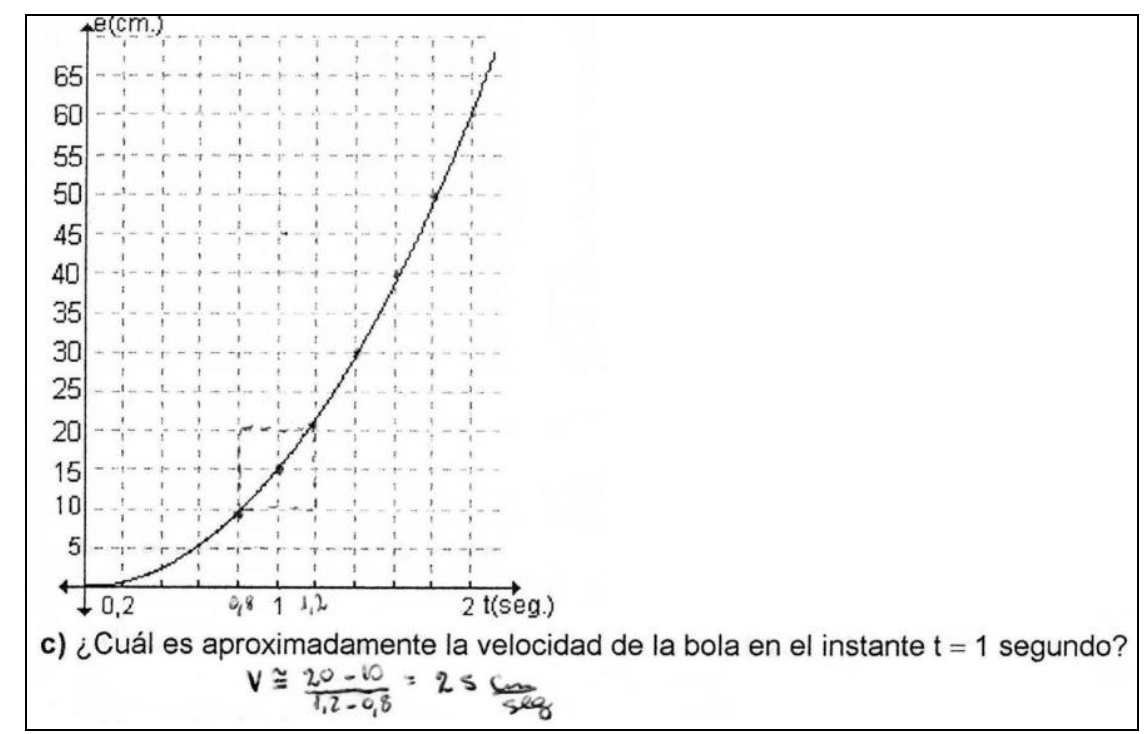

Figura 6 - Interpretación geométrica de una aproximación de la velocidad instantánea Fuente: datos de la investigación

Se observa que, para responder sobre la velocidad en el instante, no tuvieron en cuenta los intervalos propuestos, sino que realizaron su propia aproximación. Consideraron un intervalo pequeño alrededor del punto en cuestión (aspecto que, todavía, no había sido propuesto por los docentes) y determinaron la velocidad media en ese intervalo, interpretando geométricamente las distintas medidas. 
En los incisos siguientes se trabajaron las relaciones de las razones de cambio con las pendientes de las rectas secante y tangente a la curva. Esto demandó, en el contexto geométrico, el trazado de las rectas, lo que ayudó a la visualización del proceso de encontrar la recta tangente mediante aproximaciones sucesivas de rectas secantes (Figura 7).

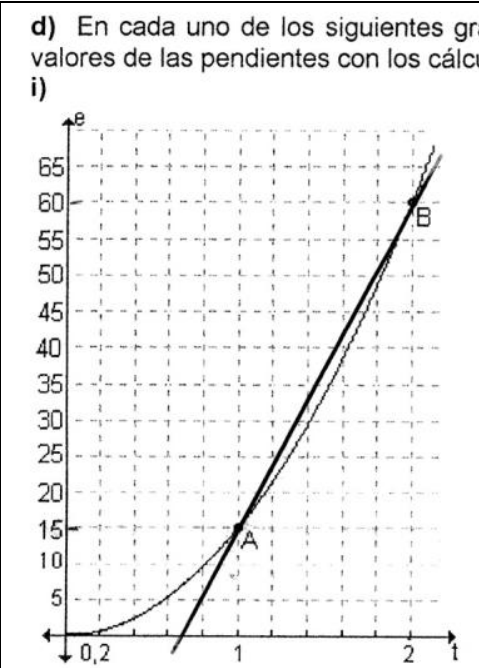

iii)
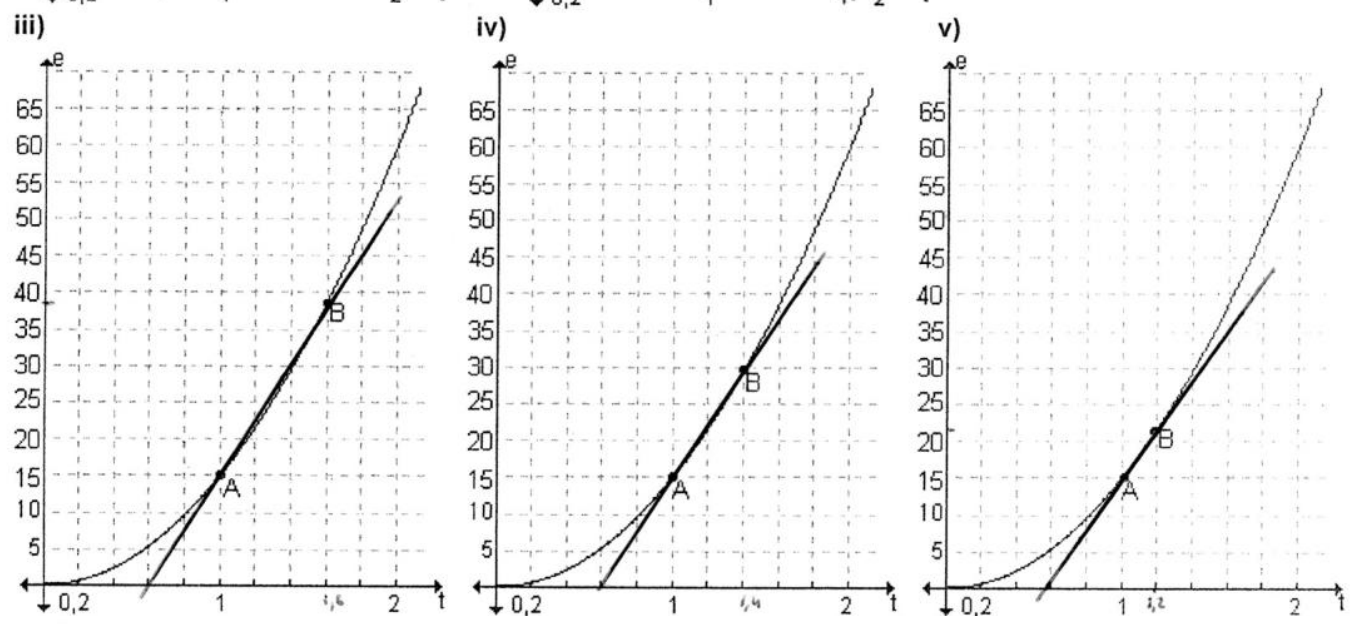
ii)

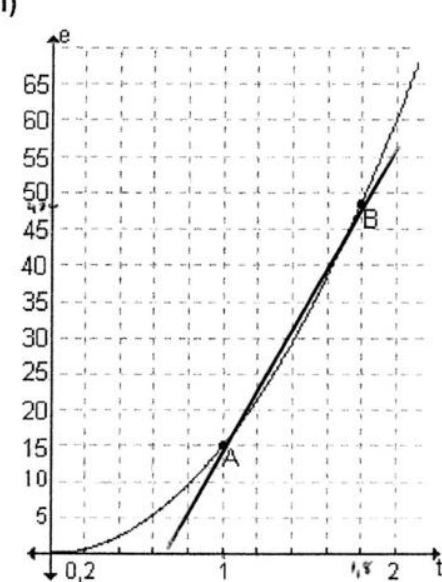

i) $m=\frac{60-15}{2-1}=45$

ii) $m=48-15=41,25$

iii) $\begin{aligned} 1,8-1 \\ =38\end{aligned}$

ia $=\frac{38-15}{1,6-1}=38,3$

iv) $M=\frac{30-15}{44-1}=37,5$

v) $m=\frac{22-15}{1,2-1}=35$

La pendiente se coleale de lo mism
forma que lo velecidod medic $r=\frac{\Delta e}{\Delta t}$ $m=\frac{\Delta e}{\Delta t}$

e) Dibuje la recta tangente a la gráfica en el punto A. Estime su pendiente ¿Qué relación existe entre la pendiente de esta recta y la velocidad pedida en c)?

La pendiente de esto recto es aproximadomente iguel a la velocidad de lo bola en $t=1$

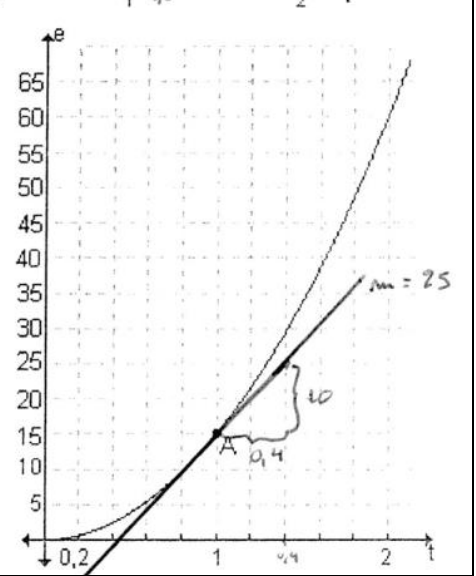

Figura 7 - Interpretación geométrica de la velocidad media y la velocidad instantánea Fuente: datos de la investigación 
En la resolución presentada en la Figura 7, se ve cómo los alumnos utilizaron recursos geométricos para estimar la pendiente. El acercamiento visual propuesto favoreció que algunos grupos descubrieran las relaciones.

La última actividad (Figura 8) tuvo carácter integrador, intentando recuperar las intuiciones, conjeturas y hallazgos que las actividades anteriores pretendieron desarrollar en los alumnos.

En el primer inciso se plantea la idea de intervalos infinitamente pequeños, además de la notación de tiende a un número $(\rightarrow \ldots)$. La estrategia central consistió en explorar qué sucede con las velocidades medias muy cerca del punto en cuestión, acercándose al mismo tanto por derecha como por izquierda. Refiere de manera intuitiva a los procesos infinitos y esperábamos que los alumnos se den cuenta de la relación con lo estudiado sobre límite.

\begin{tabular}{|c|c|c|c|c|c|c|c|c|}
\hline \multicolumn{9}{|c|}{$\begin{array}{l}\text { Actividad 2. La posición de una partícula, medida en centímetros desde cierto punto de referencia, respecto del tiempo } \\
\text { medido en segundos está dada por la ley } \mathrm{s}(\mathrm{t})=\mathrm{t}^{3} \text {. } \\
\text { a) Complete la tabla, considerando para cada valor la cantidad de lugares decimales que sean necesarios para } \\
\text { diferenciarlos entre sí. }\end{array}$} \\
\hline$t_{0} \leq t \leq t_{1}$ & $1,9 \leq t \leq 2$ & $1,99 \leq t \leq 2$ & $1,999 \leq t \leq 2$ & $\ldots \rightarrow$ & 2 & $\leftarrow \ldots$ & $2 \leq t \leq 2,001$ & $2 \leq t \leq 2,01$ \\
\hline$\underline{\Delta t}$ & 0,1 & 0,01 & 0,0001 & $\ldots \rightarrow$ & 0 & $\leftarrow \ldots$ & 0,001 & 0,01 \\
\hline$\Delta s$ & 1,141 & 0,119401 & 0,011994 & $\ldots \rightarrow$ & 0 & $\leftarrow \ldots$ & 0,012 & 0,120 \\
\hline$\frac{\Delta s}{\Delta t}$ & 11,41 & 11,94 & 11,994 & $\ldots \rightarrow$ & 12 & $\leftarrow \ldots$ & $12 ;$ & 12 \\
\hline \multicolumn{9}{|c|}{$\begin{array}{l}\text { b) Según el acercamiento realizado en la tabla, ¿qué puede decir sobre la velocidad de la partícula en } t=2 \text { ? ¿Si } \Delta t \text { es } \\
\text { infinitamente pequeño, se continuará cumpliendo esta conjetura? (o veloudod en t=2 es Aprox } 12^{\mathrm{cm} / \mathrm{seg}} \\
\text { Si Sc Sigue Cumpliendo lo Confefuro }\end{array}$} \\
\hline
\end{tabular}

Figura 8 - Acercamientos por izquierda y por derecha a la velocidad instantánea Fuente: datos de la investigación

A partir de la tabla, aproximadamente la mitad de los grupos lograron conjeturar sobre la velocidad de la partícula en el instante y el comportamiento en el caso de que los intervalos sean infinitamente pequeños. Su resolución permitió que reconozcan la necesidad de realizar el paso al límite para determinar la razón de cambio instantánea (Figura 8).

En los siguientes incisos de esta actividad se calcularon analíticamente los cambios y las razones de cambio. Las mayores dificultades se presentaron con el trabajo algebraico y la interpretación física y geométrica de los resultados. Sin embargo, varios equipos lograron observar las relaciones buscadas, como es el caso del trabajo que se presenta en la Figura 9.

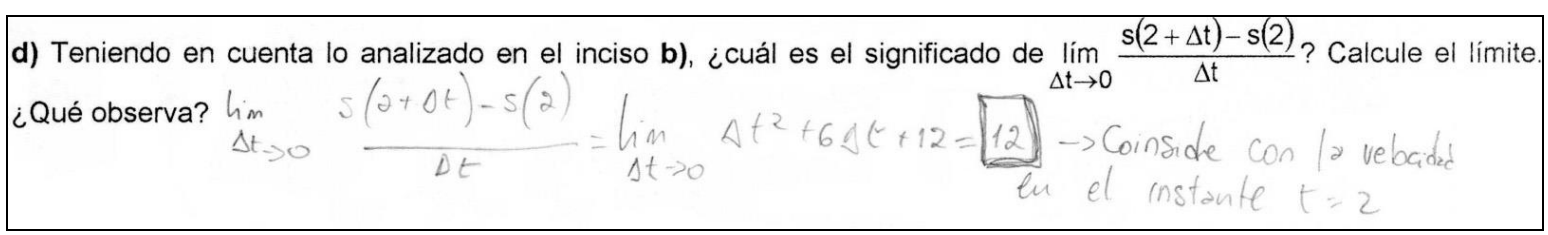

Figura 9 - Cálculo e interpretación de la razón de cambio instantánea Fuente: datos de la investigación 
La presentación de la derivada de una función en un punto en la instancia de institucionalización fue el último paso de la secuencia. De esta manera no resultó un concepto abstracto, sino que se intentó su construcción a partir de la interpretación del significado concreto, tanto físico como geométrico, de cada uno de los términos del límite planteado.

\section{Reflexiones}

Las situaciones planteadas permitieron trabajar distintos aspectos variacionales: la identificación de las funciones como relaciones entre variables en fenómenos de cambio, el análisis del comportamiento de los cambios (que da lugar a la necesidad de encontrar las leyes que describen esos cambios para poder medirlos y predecirlos), la cuantificación de los cambios, el cálculo de razones de cambio que se interpretan como la relación o comparación de los cambios de una variable con respecto a los cambios de la otra variable, la necesidad de encontrar una manera de describir y cuantificar los cambios que se dan a cada instante en una situación, la relación entre las razones de cambio con la pendiente correspondiente. La definición de derivada surgió de manera natural al final de este desarrollo, planteado a partir de la necesidad de cuantificar los cambios en un instante.

Tanto en la resolución de las actividades propuestas como en las etapas de discusión e institucionalización, los alumnos utilizaron ideas, estrategias, procedimientos relacionados al pensamiento variacional. Asimismo, plantearon argumentos y utilizaron lenguaje característico de este tipo de pensamiento.

El uso de la visualización formó parte del diseño, ya que se buscó que el alumno obtenga información relevante y explique sus conjeturas a través de las gráficas presentadas en las diferentes actividades. Se intentó, en todo momento, obtener la máxima información posible de cada una de las situaciones representadas, de manera de beneficiar el desarrollo de su capacidad de análisis. Las producciones escritas muestran que han podido manejar, en diversos grados, información visual, han logrado generar y plasmar en el papel conocimiento matemático. La exigencia de las producciones escritas y el debate oral favoreció el tratamiento y conversión entre representaciones de los registros numérico, gráfico, analítico y verbal, lo cual es imprescindible para la comprensión.

Durante las instancias de institucionalización de cada sesión se intentó que los alumnos relacionen lo trabajado en otras actividades, inclusive de sesiones anteriores, haciendo un paralelo entre distintas representaciones de un mismo registro o de registros diferentes. 
Las actividades propuestas lograron motivar a los alumnos y movilizar sus concepciones. Los alumnos sentían que eran capaces de resolverlas y el conocimiento esperado fue emergiendo tanto en las discusiones de los pequeños grupos como en la puesta en común de toda la clase.

La metodología de trabajo propuesta logró que todos se involucraran y se dispusieran a analizar las actividades planteadas. La formación de pequeños grupos favoreció también este aspecto, ya que ninguno tomó una actitud pasiva.

La secuencia cumplió con nuestras expectativas: promover la comprensión de la derivada.

Las mayores dificultades se relacionaron con el manejo de conocimientos previos necesarios, tanto a nivel conceptual como algorítmico. Por otro lado, la cantidad de nociones involucradas en la secuencia hicieron que los tiempos de trabajo, especialmente en las etapas de validación e institucionalización, fueran escasos. La misma secuencia, distribuida en más sesiones, puede llevar a mejores resultados.

En este sentido, teniendo en cuenta los problemas detectados, nos preguntamos qué acciones es posible implementar para subsanar de alguna manera las dificultades relacionadas con el bajo nivel de los conocimientos previos de nuestros alumnos, y qué modificaciones deben plantearse en las actividades propuestas de manera de optimizar el funcionamiento de la secuencia.

De todas maneras, consideramos que la resolución o, por lo menos el intento de solución de las distintas actividades, fue significativo. Los aspectos que no pudieron descubrir los alumnos en la resolución de las actividades fueron abordados luego en el debate grupal y en la institucionalización.

Creemos que el mayor valor de esta investigación radica en efectivizar una propuesta en el aula universitaria. A partir de las concepciones teóricas sobre las que se fundamenta nuestro trabajo, hemos logrado diseñar una secuencia didáctica y adaptar una metodología de trabajo a nuestro contexto particular. Si bien se han desarrollado ampliamente investigaciones relacionadas con esta temática, todavía son escasas las experiencias reales en el aula en este nivel educativo. Logramos mostrar que es posible intentar cambios en la enseñanza buscando el trabajo comprometido de los alumnos.

Estamos convencidas de que, con nuestro trabajo, facilitamos que produzcan conocimiento matemático, reflexionen sobre sus producciones y generen teoría sobre dicho conocimiento. Todo esto deriva necesariamente en aprendizajes significativos. 


\section{Referencias}

ARTIGUE, M. La enseñanza de los principios del cálculo: problemas epistemológicos, cognitivos y didácticos. In: ARTIGUE, M.; DOUADY, R.; MORENO, L.; GÓMEZ, P. (Ed.). Ingeniería didáctica en educación matemática. México: Grupo Editorial Iberoamérica, 1995. p. 97-140.

AZCÁRATE, C.; BOSCH, D.; CASADEVALL, M.; CASELLAS, E. Cálculo diferencial e integral. España: Síntesis, 1996.

BUENDÍA, G.; ORDÓÑEZ, A. El comportamiento periódico en la relación de una función y sus derivadas: significados a partir de la variación. Revista Latinoamericana de Investigación en Matemática Educativa, México, D. F., v. 12, n. 1, p. 7-28, mar. 2009.

CANTORAL, R.; FARFÁN, R.; CORDERO, F.; ALANÍS, J.; RODRÍGUEZ, R.; GARZA, A. Desarrollo del pensamiento matemático. México: Trillas, 2003.

CANTORAL, R., MOLINA, J.; SÁNCHEZ, M. Socioepistemología de la Predicción. In: LEZAMA, J.; SÁNCHEZ, M. y MOLINA, J. (Ed.). Acta Latinoamericana de Matemática Educativa. México: Comité Latinoamericano de Matemática Educativa, 2005. p. 463-468.

CANTORAL, R.; MONTIEL, G. Funciones: Visualización y Pensamiento Matemático. México: Pearson Education, 2001.

DOLORES, C. Una introducción a la derivada a través de la variación. México: Grupo Editorial Iberoamérica, 1999.

DOLORES, C. La derivada y el Cálculo. Una mirada sobre su enseñanza por medio de los textos y programas. In: DOLORES, C.; MARTÍNEZ, G.; FARFÁN, R.; CARRILLO, C.; LÓPEZ, I.; NAVARRO, C. (Ed.). Matemática Educativa: Algunos aspectos de la socioepistemología y la visualización en el aula. México: Ediciones Díaz de Santos, 2007a. p. 169-204.

DOLORES, C. Elementos para una aproximación variacional a la derivada. México: Universidad Autónoma de Guerrero y Ediciones Díaz de Santos, 2007b.

DUVAL, R. Registros de representación semiótica y funcionamiento cognitivo del pensamiento. In: HITT, F. (Ed.). Investigaciones en Matemática Educativa II. Traducción de: Registres de représentation sémiotique et functionnement cognitif de la pensée. México: Grupo Editorial Iberoamérica, 1998. p. 173-201. (Annales de Didactique et de Sciences Cognitives. Vol. 5 (1993)).

DUVAL, R. Eight problems for a semiotic approach in mathematics education. In: RADFORD, L.; SCHUBRING, G.; SEEGER, F. (Ed.). Semiotics in mathematics education: epistemology, historicity, classroom, and culture. Rotterdam: Sense Publishers, 2008. p. 39-62.

TESTA, Z. Procesos de resignificación del valor numérico de la función derivada segunda: Un estudio en el sistema escolar uruguayo. 2004. 333 f. Tesis (Maestra en Ciencias en Matemática Educativa) - Centro de Investigación en Ciencia Aplicada y Tecnología Avanzada del IPN, México, 2004.

WENZELBURGER, E. Didáctica Cálculo diferencial. México: Grupo Editorial Iberoamérica, 1993.

Submetido em Novembro de 2012. Aprovado em Abril de 2013. 